\title{
Parking Management System using Image Processing and Distributed Approach
}

\author{
Prof. S.P. Bholane', Shantanu Kotambkar ${ }^{2}$, Ajinkya Lakade ${ }^{2}$, Kunal Mande', Tanmay Deshmukh ${ }^{2}$ \\ Assistant Professor, Department of Computer Engineering, Sinhgad College of Engineering, Pune, India ${ }^{1}$ \\ Student, Department of Computer Engineering, Sinhgad College of Engineering, Pune, India ${ }^{2}$
}

\begin{abstract}
Vehicle parking in today's date has turned into a noteworthy issue in urban territories, because of the absence of parking facilities and poor management. Problems emerging from the absence of parking facilities and poor administration incorporate movement clog, expanded contamination, expanded utilization of fuel.To relieve these issues, a model has been produced with the android application, appropriated frameworks, image processing. The prototype with the help of an android application gives the information regarding the availability of car parking to the user directly. Delivering User the information regarding the parking space availability will thus reduce the time, fuel and efforts invested in search of a parking space. This will also lead to a reduction in traffic congestion, ultimately leading to less pollution. A database maintained for the availability of parking space can be used for better management of parking spaces. For detection of car parking spaces, image processing is helpful which will determine the parking availability. This prototype hence, will reduce manpower needs and increase the flexibility and security. The image processing at the local server level and only providing the required information to the central server thus optimizes the process followed by this prototype. Thus, a distributed parking prototype is developed to mitigate the problems faced by a common man and in the process conserving fuel and helping reduce global warming, pollution as well.
\end{abstract}

Keywords: Image processing, car park occupancy, object detection, information system.

\section{INTRODUCTION}

The model with the assistance of an android application gives the data in regards to accessibility of available parking details to the client directly. The user knows the parking spot accessibility, in this way lessening the time, fuel and endeavors put resources into the search for a parking space. This will likewise prompt the user to look for parking spaces easily. A database in which the accessibility of parking spot is overseen helps maintain real-time availability data. For recognition of vehicle parking spots, real-time processing of surveillance video will help decide the parking accessibility. This model hence, will decrease labor needs and increment adaptability and security. The image processing is done at the local server level and just the required data is given to the central server, thus diminishing the enhancing the procedure and expounding the disseminated approach took after by this model. Thus, a appropriated parking model is created to moderate the issues confronted by a typical man and in the process monitoring fuel and decreasing global warming, air pollution.

The project "Parking Management System using Image Processing and Distributed Approach" is made with an outlook to solve a civic issue of free parking space management and employ them in urban areas. The project considers a distributed approach to solving the issues, thus minimizing manpower needs and maximizing the optimal efficacy of the system.

Parking Management has been an issue of concern in today's date due to the increasing ratio of personal vehicles to parking spaces. The project works on the concepts of distributed processing, image processing, android application development and web application development is aimed to increase the productivity of parking system. The parking slots are fulfilled according to the user requirement, thus ensuring optimal use of parking space and user's ease of access.

\section{RELATED WORK}

This section provides a brief overview of relevant literature on parking availability detection, including supporting information on related work in Real-time Data Extraction, Decision Module, Driver's guidance.

\subsection{Real-time Data Extraction.}

Provided that the purpose of this approach is to ensure that system should be adapted for multiple parking dispositions, the aim is to introduce a real-time approach for data extraction and moving car detection. Since cameras are used to provide fixed scene video frames, it is proposed to detect the car in its motion. This allows to allocate the car a suitable parking slot and update the parking system count of parking slots.

The first phase is to interpret a base frame for image processing. Whenever a car enters the frame, we extract the motion of the cars in the fixed scene. Providing the base frame as the initial condition, we can opt to the implementation of adaptive background subtraction 
algorithm that adaptively eliminates the background of the parking video frame and consequently, detects and extracts the car in its motion. The first approach involves extraction. Secondly, the number of slots correspond to the detected cars. So as to update the slot vacancy data in our central server and also notify the user regarding available vacant slots.

\subsection{Parking Event Detection and Update in Database}

Previous work that has been carried out in the area of car motion detection. This section focuses on the back-end event detection and updates to the central database. As soon as the car is detected in its motion using background subtraction algorithm, an event is triggered and an update to the database is sent. Another such event is triggered after every 15 frames are captured and processed to assist and ensure timely update of the database. Along with this, a user triggers an event as soon as an update is requested by the user. Thus, the timely and well-maintained database is updated.

\subsection{Integrated Approach in the Design of Car Park Occupancy}

In large parking areas such as those at mega shopping malls or stadiums, drivers always have difficulty to find vacant car park lots especially during peak periods or when the parking lots are almost full. A solution to reduce the drivers' searching time for vacant car-park lots will greatly save time, reduce cost and improve the traffic flow in the car park areas. In this approach, a research project which was developed to acquire car-park occupancy information using an integrated approach of image processing algorithms is used. The motivation for developing this system came from the fact that minimum cost is involved because image processing technique is used rather than sensor-based techniques. Security surveillance cameras which are readily available in most car parks can be used to acquire the images of the car park. This solution is much cost effective than installing the sensor on each parking lot.

A wide range of stopping direction frameworks have been created and intended to abbreviate the looking time for empty parking areas. This is particularly essential for drivers who need to scan for accessible auto parks amid pinnacle hours or when the auto parks are full. Searching for parking space inhabitance data, for the most part, fall into four classifications - counter-based, wired-based, remote based and picture based frameworks.

\section{III.SYSTEM OVERVIEW}

The main objective of this proposed system is to provide a real-time solution for the ongoing parking problems especially in the metropolitan cities where searching for parking space is a major problem. This proposed solution will easily adapt to various parking system.

There are several approaches to solving such problems but those require heavy manpower duty or heavy sensor equipment which proves to be rather costly. This system will not only provide an efficient solution to such problems but also will help reduce the pollution and keeping the environment safe.

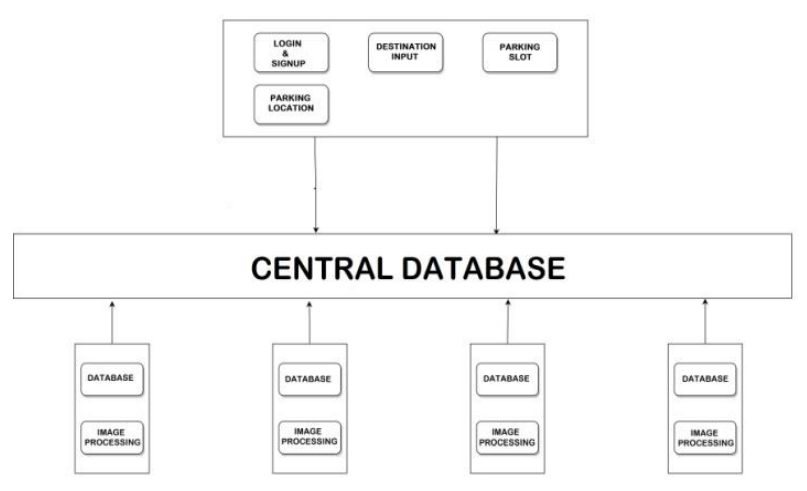

Fig.01. Architectural Overview

There are three modules to implement this system. Firstly, the image processing module where at various parking system the number of parking slots available will be calculated with the help of image processing. Secondly, the central server where the actual data would reside. The data from image processing is sent to the central server. The third module consists of android application where the individual user can access the data at the central server. The proposed system consisting of these modules will help the user at any location to search for available parking slot via various parking options in the android application. The user will get to locate available parking spaces nearest to their location.

\subsection{Real-time data extraction using Image processing}

In this module, some set of algorithms is applied to realtime parking system through a surveillance camera. This surveillance camera will enable this system to perform image processing on live video tracking of a specific parking system. Initial frames from the video are recorded with all empty parking spaces empty as a reference point. This method is called offline configuration. After this method, image frames with vehicles parked at the specific parking slot are recorded and using background subtraction method, these frames are subtracted to obtain the desired result. A contour is drawn which is nothing but a rectangular boundary alongside the vehicle. This contour helps to determine whether a parking spot is occupied or vacant. The number of parking slots available is calculated and these data is stored in the database at the central server.

\subsection{Distributed Approach}

This section discusses the distributed approach used in the Parking Management System. The distributed approach consists of a central Database and several local Database servers, each handling an individual parking system. The central database, handle multiple local servers and thus, serves as the main information provider to the user. The 
user posts a query regarding a particular parking system to the central server, which in turn retrieves the information from the appropriate local database and then sends the data to the user. This enables lesser congestion of network, provides better security of data and optimizes the Parking System management using Distributed approach.

\subsection{Android Applications}

The Android application enables the user to search for The proposed framework gives an alternative way to parking spaces near their specified location. The user will traditional parking management system, to deal with get the number of parking systems on the server and will identification of opportunities of parking spots. This be able to access the available parking slot in the specified parking system. Android application will also keep Login/Sign-Up info to specify parking spots for the specified user. Android Application gives a real-time solution to search for parking in the crowded cities among various options.

As the name suggests, "Parking Management System using Image processing and Distributed approach", the system uses Image processing, Distributed Network consisting of a central and local database, along with an android application for user convenience. The image processing module performs the major task of detecting and assessing the count of vacant parking slots. This count is updated in a local database at regular intervals and whenever is an event trigger database access requirement. This local data is fetched from the central server when the user requests for information about the corresponding parking system. The android application provides functionality such as User login and sign-up. The android application displays all nearby parking locations along with their distance from the user. The user can select the parking location and view the available parking slot.

\section{IV.ALGORITHM}

\section{Image processing Algorithm}

The image processing algorithm is used to detect vehicles at the parking slot and to determine whether the parking spot is empty. The following algorithm is implemented using OpenCV libraries.

1) Initial setup of parking system requires and image frame which consists of all empty parking slots in the parking system. This frame is stored in a matrix as a reference to the parking system. This frame is referred as initial frame.

2) When a vehicle arrives, the image frames with the vehicle at a specific parking slot is gathered. These frames denote that a vehicle arrives in the frame boundary.

3) Subtract the initial frame from the frame where the vehicle is in parking space. Store this difference in a matrix.

4) Use all the frames of the vehicle arriving to draw a contour alongside the boundary of the vehicle. Store this contour in a list. Predefine a threshold value for the vehicle size according to the image frame.

5) If the contour size is in the range of threshold value then increment the count for a number of vehicles parked.
6) Calculate the number of parking available and store this data in the database at the central server.

This algorithm runs at the every parking system and collects data from all parking system and store it in the database at the central server.

\section{CONCLUSION}

approach comprises of distinguishing empty parking spots by means of a surveillance camera. The framework likewise gives drivers' information assistance. This module gives a continuous refresh of parking slots to drivers through cellphone application with a specific end goal to guide them and encourage the errand of finding an accessible parking slot.

The proposed system provides a vision-based approach for detecting vacancies of parking spaces for a given context. This approach of the module provides real-time updates of parking spaces to drivers via mobile phone application in order to guide them and facilitate the task of locating an available parking space

\section{ACKNOWLEDGMENT}

We want to express our earnest appreciation towards our guide Prof. S. P. Bholane for his significant direction and supervision that helped us in our exploration. He has constantly motivated us to investigate new ideas and seek after more up to date examine issues. We credit our venture commitment to him. By and large, we likewise want to thank our review committee members Prof. A. R. Joshi and Prof. S. S. Pawar for their time, proposals, and for thoughtfully consenting to be on our review committee, and continually making themselves accessible. We can't express gratitude toward them enough.

\section{REFERENCES}

[1] Masmoudi, Imen, et al. "Trajectory Analysis for Parking Lot Vacancy Detection System.” IET Intelligent Transport Systems (2016).

[2] Masmoudi, Imen, et al. "Architecture of Parking Lots Management System for Drivers' Guidance." Systems, Man, and Cybernetics (SMC), 2015 IEEE International Conference on. IEEE, 2015

[3] Bong, D.B.L., K.C. Ting, and K.C.Lai." Integrated approach in the design of car park occupancy information system(COINS).'”IAENG International Journal of Computer Science 35.1 (2008): 7-14.

[4] Suhr, Jae Kyu, and Ho Gi Jung. "Sensor fusion-based vacant parking slot detection and tracking." IEEE Transactions on Intelligent Transportation Systems 15.1 (2014): 21-36.

[5] Tschentscher, Marc, et al. "Scalable real-time parking lot classification: An evaluation of image features and supervised learning algorithms." 2015 International Joint Conference on Neural Networks (IJCNN). IEEE, 2015.

[6] Momin, B. F., and S. M. Kumbhare. "Vehicle detection in video surveillance system using Symmetrical SURF." Electrical, Computer and Communication Technologies (ICECCT), 2015 IEEE International Conference on. IEEE, 2015. 